\title{
Modélisation probabiliste de la disposition dans le plan horizontal des racines adventives autour de la tige de maïs (Zea mays L)
}

\author{
J Chadœuf 1, S Pellerin 2, F Tricot 2

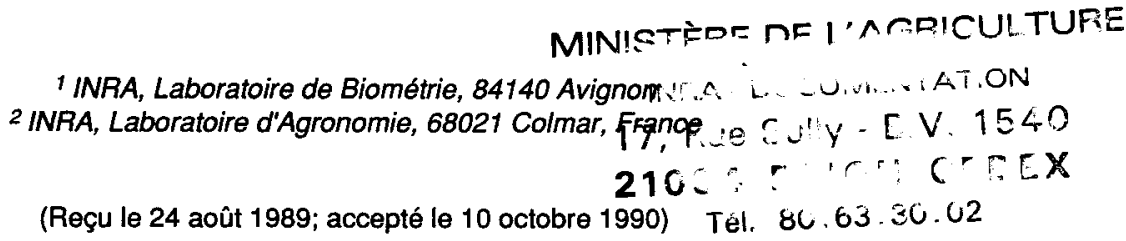

Résumé - La disposition spatiale des racines primaires du maïs autour de la tige est décrite par un modèle statistique basé sur un processus de Gibbs et un processus markovien. L'hypothèse de base sur laquelle repose le modèle porte sur la définition d'un potentiel d'interaction entre paires de racines situées sur un même entre-nœud et sur 2 entre-nœuds successifs. La disposition des racines sur un entre-nœud donné est alors dépendante d'un potentiel global défini comme la somme de ces potentiels d'interaction et d'un potentiel principal, lié au nombre de racines de l'entrenœud. II nous permet ainsi de résumer et de quantifier les observations angulaires sur les entre-nceuds $2-6$ au travers d'un faible nombre de paramètres significatifs (23). L'étude effectuée a montré une bonne adéquation des résultats du modèle avec les données observées. On montre que les positions des racines situées sur un même entrenœud comme celles situées sur 2 entre-nœuds successifs ne sont pas indépendantes. Le potentiel d'interaction de 2 racines situées sur 2 entre-nœuds successifs est d'environ $75 \%$ du potentiel d'interaction entre 2 racines du même entre-nœud tant en intensité maximale qu'en portée.

Zea mays / disposition des racines / modélisation

Summary - Probabilistic modeling of primary root arrangement in the horizontal plane around the maize stem. The spatial arrangement of the maize roots around the stem in the horizontal plane is described by way of a statistical model. Based upon a Gibbs process and a markovian hypothesis, it allows us to summarize and quantify the angular observations on internodes 2-6 through a small number of significant parameters (23). The basic assumption of the model is that an interaction potential between 2 roots on a single internode and between 2 roots on 2 successive internodes can be defined. For 2 roots on the same internode (respectively 2 successive internodes) separated by an angle $\theta$, the interaction potential is defined as

$$
\begin{gathered}
\mathrm{V}(\theta)=\mathrm{b}\left[\cos \left(\pi \frac{\theta}{\mathrm{a}}\right)+1\right] \\
\left(\text { respectively } \mathrm{V}(\theta)=\mathrm{d}\left[\cos \left(\pi \frac{\theta}{\mathrm{c}}\right)+1\right]\right)
\end{gathered}
$$

if $|\theta|<a(r e s p<c), V(\theta)=0$ if $|\theta| \geq a$ (resp $\geq c)$. The parameters $a, b, c$ and d depend on the internodes. The parameter a (resp c) characterizes the range of the interaction, $b$ (resp d) its intensity. The disposition of the roots of an internode is then dependent upon the total potential, defined as the sum of the interaction potentials and of a principal term depending on the number of roots on the internode. Simulation studies show a good fit between the results of the model and the actual data. This is the case for the distribution of the number of roots on each internode (fig 3 and table III). Angular distributions between actual and simulated data have also been compared. These are the angle between 2 successive roots on a single internode (figs 4 and 5) and the radio $\alpha / \beta$ where $2 \beta$ is the angle between 2 successive roots on a single internode and $\alpha$ is the angle of the bissectrix of $\beta$ and a root on the next internode whose position is between the 2 preceeding roots.

It is shown that both roots on the same internode and on 2 successive internodes are interacting (table II). More particularly, the interaction potential between 2 roots on successive internodes is about $75 \%$ of the interaction potential between 2 roots on the same internode. 


\section{INTRODUCTION}

La modélisation de l'absorption d'eau et d'éléments minéraux par une culture est actuellement limitée par la difficulté de prendre en compte les caractéristiques géométriques du système racinaire. Celui-ci a généralement une morphologie arborescente complexe sujette à de nombreuses variabilités et difficile à décrire in situ autrement que par des méthodes destructives. Cela souligne l'intérêt des modèles dits d'architecture qui simulent en 3 dimensions la mise en place du système racinaire par initiation, allongement et ramification de petits segments représentant les racines (Lungley, 1973; Diggle, 1988; Pagès et Ariès, 1988). Une description de la géométrie des systèmes racinaires est un préalable nécessaire à leur mise en œuvre.

Le système racinaire du maïs est constitué de racines séminales situées à la base du mésocotyle et de racines adventives néoformées apparaissant à la base de la tige sur les entre-nœuds successifs. Les trajectoires des racines adventives dans le sol ont été étudiées (Tardieu et Pellerin, 1990). Pour compléter la description géométrique du système racinaire adventif primaire, il était nécessaire d'étudier la disposition de ces racines autour de la tige. Une première analyse a déjà été réalisée (Pellerin et al, 1989). Contrairement aux feuilles, les racines adventives ne sont pas disposées autour de la tige selon un modèle simple. Leur disposition n'est cependant pas totalement aléatoire. On a pu mettre en évidence quelques règles de disposition. Ainsi lorsqu'une racine d'un entre-nœud apparaît dans l'angle formé par 2 racines de l'entre-nœud précédent, elle occupe dans la majorité des cas une position proche de la bissectrice. Cette première analyse restait cependant partielle car basée sur l'étude séparée de quelques configurations particulières. Dans une optique de modélisation de l'architecture du système racinaire il était nécessaire d'étudier globalement la distribution statistique de ces racines autour de la tige et de pouvoir faire des simulations. C'est pourquoi nous proposons ici un modèle probabiliste de description du nombre et de la position angulaire des racines adventives autour de la tige. Basé sur des notions de distance angulaire, le modèle permet de quantifier les relations entre racines et entre les différents entre-nœuds.

\section{MATÉRIEL ET MÉTHODES}

\section{Données}

Les données ont été recueillies sur 63 pieds de maïs (variété Déa) cultivés selon les normes de la grande culture sur sol limoneux profond (argile 20,7\%, limon fin $31,1 \%$, limon grossier $38,2 \%$, sable fin $5,2 \%$, sable grossier 2,9\%, pour l'horizon 0-30 cm - Domaine expérimental INRA Colmar). La nomenclature utilisée pour désigner les entre-nœuds successifs est celle proposée par Girardin et al (1986). Le permier entrenœud portant des racines adventives est nommé entre-nœeud 2. Sur chacun des pieds on a compté le nombre de racines sur les entre-nœuds $E_{2}$ à $E_{6}$ et mesuré leur position angulaire dans un plan horizontal grâce à un dispositif micrométrique mobile en rotation permettant de déplacer un repère sur les racines successives. Ce dispositif a été décrit en détail dans l'article précédent (Pellerin et al, 1989). Pour chaque pied une racine de l'entre-nœud 2 était arbitrairement choisie comme référence. Quelques pieds observés sont présentés schématiquement en projection horizontale dans la figure 1. Comme cela a été indiqué précédemment, on observe que la répartition des racines n'est pas quelconque. Sur un entre-nœud donné, elles se

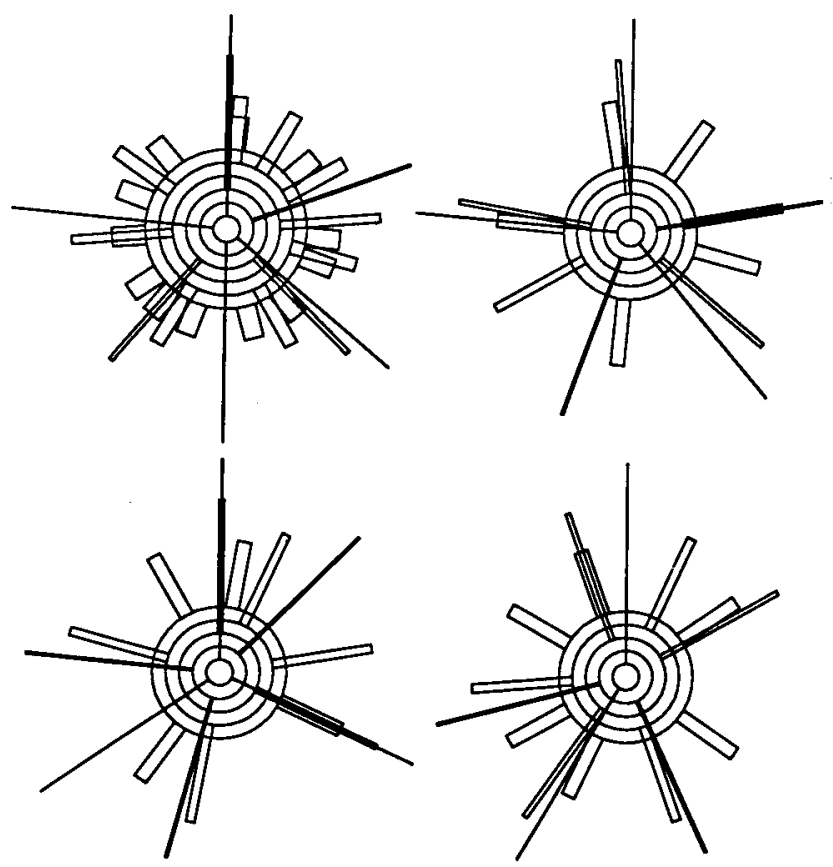

Fig 1. Représentation schématique des dispositions observées. Chaque entre-nceud est représenté par un cercle, de diamètre proportionnel à son niveau.

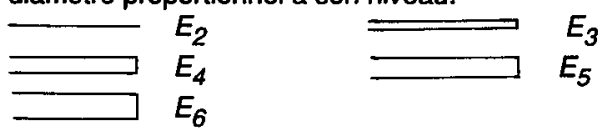


répartissent de façon relativement régulière autour du pied. Elles ont aussi tendance à se placer entre les racines de l'entre-nœud précédent.

\section{Modèle}

\section{Hypothèses biologiques}

L'organogenèse des racines adventives a été décrite par plusieurs auteurs (Girardin et al, 1987; Hoppe et al, 1987). Il y a d'abord formation d'ébauches à la périphérie de la tige par dédifférentiation de cellules du parenchyme sous-cortical. L'origine des racines adventives est donc très superficielle et des coupes transversales de la base de la tige n'ont mis en évidence aucune localisation particulière de ces ébauches par rapport à la structure vasculaire de la tige. Ultérieurement, ces ébauches s'allongent et percent la gaine de la feuille couvrant l'entre-nœud dont elles sont issues. L'apparition des ébauches, puis leur allongement se fait successivement des entre-nœuds inférieurs vers les entre-nœuds supérieurs. On peut donc considérer a priori que les racines en formation sur un entre-nœud sont en interaction avec les autres racines du même entre-nœud, et avec les racines des entre-nceuds inférieurs.

Dans le modèle, nous supposerons que la distribution des racines sur un entre-nœud donné (nombre et disposition) dépend uniquement du numéro de l'entrenœud et de la distribution des racines sur l'entre-nœud précédent. Ceci correspond donc à l'hypothèse d'un double jeu de dépendances : au niveau d'un entrenœud d'une part, d'un entre-nœud au suivant d'autre part. Les éventuelles liaisons à plus longue distance sont négligées.

\section{Modélisation probabiliste}

Pour $E_{p}$ entre-nceud numéro $p$, nous notons $I_{p}$ le nombre de racines primaires présentes : $R_{p}=\left\{\left(R_{p, j}\right)\right.$, $\left.j=1, \ldots, I_{p}\right\}$ est l'ensemble de ces racines et $\lambda_{p}=$ $\left\{\left(\lambda_{p, j}\right), j=1, \ldots, I_{p}\right\}$ les positions angulaires de ces racines. D'après la convention mentionnée plus haut, la position angulaire de la première racine est nulle, soit $\lambda_{2,1}=0$. Reprenant les hypothèses du paragraphe précédent, nous supposerons négligeables les liaisons au-delà de 2 entre-nœuds successifs. Au niveau de chaque entre-nœud, les positions des différentes racines vont être décrites par un modèle de Gibbs (Stoyan et al, 1987) dépendant de l'entre-nœud précédent. Nous supposerons de plus que seules interviennent les liaisons entre paires de racines. Pour cela, nous modélisons ces liaisons entre 2 racines de positions angulaires $\theta_{1}$ et $\theta_{2}$ exprimées en radians au travers d'un potentiel du type $V\left(\theta_{1}-\theta_{2}\right)=b\left(\cos \left(\pi \theta_{1}\right.\right.$ $\left.-\theta_{2}(a)+1\right)$ si $\left|\theta_{1}-\theta_{2}\right| \leq a$, et $V\left(\theta_{1}-\theta_{2}\right)=0$ sinon, $a$ et $b$ sont des paramètres réels dépendant du rang des entre-nœuds des 2 racines considérées. Ils vérifient 0 $\leq a \leq \pi$ et $b \leq 0$. La courbe correspondant à ce potentiel est tracée dans la figure 2. Le paramètre a correspond à la distance angulaire maximale au-delà de laquelle il n'y a plus interaction, $2 b$ correspond à l'intensité maximale de cette interaction.

\section{Entre-nœud 2}

Les racines de l'entre-nœud 2 sont les premières racines adventives apparaissant à la base de la tige. Le potentiel global, noté $W\left(R_{2}\right)$, correspondant à l'ensemble des racines de l'entre-nœud 2 est considéré comme égal à la somme des potentiels d'interaction entre paires de racines et d'un potentiel principal $e l_{2}$ proportionnel au nombre de racines :

$$
W\left(R_{2}\right)=\sum_{1 \leq i \leq j \leq I_{2}} \vee\left(\lambda_{2, i}-\lambda_{2, j}\right)+e l_{2}
$$

L'idée du modèle de Gibbs est d'associer à chaque configuration une probabilité de réalisation liée à la valeur de son potentiel. Une configuration sera d'autant plus fréquente que son potentiel est élevé (Stoyan et al, 1987). Si $S$ et $T$ sont 2 configurations données, le rapport de leurs probabilités sera donné par:

$$
\frac{P(S)}{P(T)}=\exp (W(S)-W(T))
$$

La probabilité $P\left(R_{2}\right)$ d'observer l'ensemble $R_{2}$ est donc liée à la valeur du potentiel $W\left(R_{2}\right)$ par :

$$
P\left(R_{2}\right)=\exp \left(W\left(R_{2}\right)\right) / Z
$$

où Z est une constante de normalisation :

$$
\begin{gathered}
Z=\sum_{I_{2}=1}^{\infty} \int_{\lambda_{2,2} \in[0,2 \pi]} \cdots \\
\int_{\lambda_{2, I_{2}} \in\left[\lambda_{\left.2, I_{2}-1,2 \pi\right]}\right.} \exp \left(W\left(R_{2}\right)\right) d \lambda_{2,2} \ldots d \lambda_{2, I_{2}}
\end{gathered}
$$

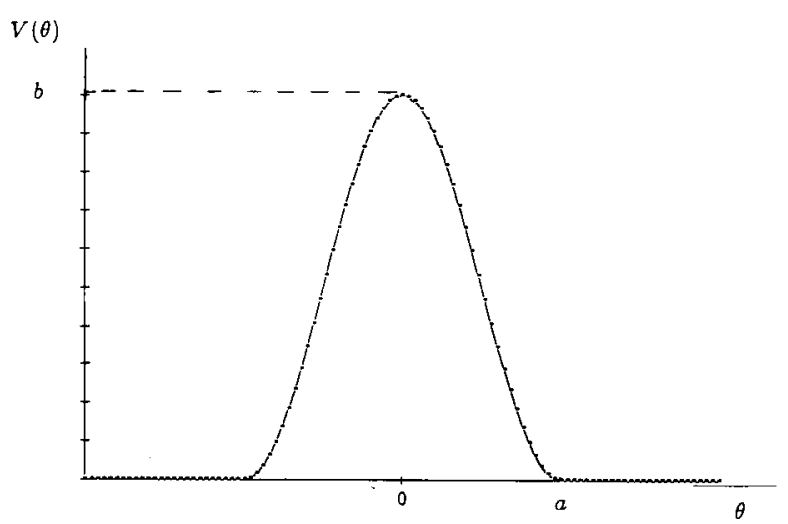

Fig 2. Forme du potentiel d'interaction entre 2 racines séparées d'un angle $\theta$. Il est donné par $V(\theta)=b(\cos (\pi \theta / a)+1)$ pour $|\theta| \leq a, V(\theta)=0$ sinon. 
On peut remarquer que, dans le calcul du potentiel, - si $b=0$, la valeur du potentiel est constante (égale à $e l_{2}$ ) quel que soit l'angle entre 2 racines. Toutes les configurations seront équiprobables (disposition totalement aléatoire des racines, c'est-à-dire une répartition uniforme et indépendante entre 0 et $2 \pi$ des angles des racines) pour un nombre de racines donné.

- si $b<0$, le potentiel entre 2 racines, qui sera alors négatif, sera d'autant plus grand (c'est-à-dire proche de 0 ) qu'elles sont plus éloignées. Les configurations les plus régulières seront les plus probables. Par exemple, pour 3 racines, la position la plus probable sera celle où 2 racines successives forment un angle de $120^{\circ}$.

- si $b>0$, le potentiel entre 2 racines sera d'autant plus grand que les racines sont proches. Les configurations où toutes les racines sont proches l'une de l'autre seront les plus probables. Cependant, un tel modèle n'est défini que pour un nombre maximal fini de racines. Par la suite nous supposerons $b<0$.

Conditionnellement à $l_{2}$, on a également :

$$
\begin{gathered}
P\left(R_{2} / I_{2}\right) \\
=\frac{\exp \left(W\left(R_{2}\right)\right)}{\int_{\lambda_{2,2} \in[0,2 \pi]} \cdots \int_{\lambda_{2, I_{2}} \in\left[\lambda _ { 2 , I _ { 2 } - 1 , 2 \pi ] } \operatorname { e x p } \left(W\left(R_{2}\right) d \lambda_{2,2} \ldots d \lambda_{2, I_{2}}\right.\right.}}
\end{gathered}
$$

\section{Entre-nœuds supérieurs}

Notons plus généralement $\left(\lambda_{p, j}\right) 1 \leq j \leq l_{p}$ les positions des racines $R_{p}$ de l'entre-nœud $p$. Le potentiel total noté $W\left(R_{p}\right)$ au niveau de l'entre-nœud $E_{p}$ est défini par la somme de 3 potentiels, $W\left(R_{p}\right)=W 1\left(R_{p}\right)+W 2$ $\left(R_{p}, R_{p-1}\right)+e I_{p}$ avec

$$
-W_{1}\left(R_{p}\right)=\sum_{1 \leq i<j \leq I_{p}} V\left(\lambda_{p, i}-\lambda_{p, j}\right) \text { potentiel total d'inte- }
$$

raction des racines de l'entre-nœud $p$. Le potentiel choisi est du même type que pour l'entre-nœud 2 ,

$$
\begin{aligned}
& V\left(\theta_{1}-\theta_{2}\right)=b\left(\cos \left(\pi \theta_{1}-\theta_{2} / a\right) \text { si }\left|\theta_{1}-\theta_{2}\right| \leq a, V\left(\theta_{1}-\theta_{2}\right)\right. \\
& =0 \text { sinon } \\
& \quad-W_{2}\left(R_{p}, R_{p}-1\right)=\sum_{1 \leq i \leq I_{p}, 1 \leq j \leq I_{p-1}} V^{\prime}\left(\lambda_{p, i}-\lambda_{p-1, j}\right)
\end{aligned}
$$

potentiel total dinteraction entre racines de l'entrenœud $p$ et de l'entre-nœud $p-1$, avec de façon similaire à $V$,

$V^{\prime}\left(\theta_{1}-\theta_{2}\right)=d\left(\cos \left(\pi\left(\theta_{1}-\theta_{2}\right) / c\right)+1\right)$ si $\left|\theta_{1}-\theta_{2}\right| \leq c$, $V^{\prime}\left(\theta_{1}-\theta_{2}\right)=0$ sinon.

- el potentiel principal proportionnel au nombre de racines de $E_{p}$.

Si $d=0, V^{\prime}\left(\theta_{1}-\theta_{2}\right)=0$, quelle que soit la valeur de l'angle entre 2 racines des 2 entre-nœuds $E_{p}$ et $E_{p-1}$. Les dispositions des racines des 2 entre-nœuds sont indépendantes. Si $b=0, V\left(\theta_{1}-\theta_{2}\right)=0$ quelle que soit la valeur de l'angle entre 2 racines de $E_{p}$. La disposition des racines sur $E_{p}$ ne dépend que de la distribution sur $E_{p-1}$. La probabilité d'observer les positions $\left(\lambda_{p, j}\right)$ connaissant l'état de l'entre-nœud $E_{p-1}$ est :

$$
\begin{gathered}
P\left(R_{p} / R_{p-1}\right)=e^{W\left(R_{p}\right)} / Z \\
Z=\sum_{I_{p}=0}^{\infty} \int_{\lambda_{p, 1} \in[0,2 \pi]} \cdots \\
\int_{\lambda_{p, I_{p}} \in\left[\lambda_{\left.p, I_{p}-1,2 \pi\right]}\right.} e^{W\left(R_{p}\right)} d \lambda_{p, 1} \ldots d \lambda_{p, I_{p}}
\end{gathered}
$$

\section{Pour un pied donné}

Si nous considérons l'ensemble des entre-nceuds mesurés, la probabilité d'observer l'ensemble des racines, $\{((R p, j), 1 \leq j \leq l p), 2 \leq p \leq 6\}$, est donnée par le produit des probabilités conditionnelles :

$$
P=P\left(R_{6} / R_{5}\right) P\left(R_{5} / R_{4}\right) \ldots P\left(R_{2}\right)
$$

et dépend de 23 paramètres

-3 paramètres pour $E_{2}: a, b$ et $e$

- 5 paramètres pour chacun des entre-nœuds suivants : $a, b, c, d$ et $e$.

\section{Estimation}

L'estimation des paramètres a été faite par la méthode du maximum de vraisemblance (Dacunha-Castelle et Duflo, 1983). Les intégrales ne pouvant être calculées analytiquement et pouvant comporter de 2 à 20 variables d'intégration, elles ont été évaluées par la méthode de Monte-Carlo. De plus, la série $Z$ converge rapidement (les termes étant en $(2 \pi)^{n} / n ! e^{A n}+b n^{2}$ avec b $\leq 0)$ ce qui permet de se limiter aux 20 premiers termes de la série dans la gamme de paramètres rencontrée.

On a testé l'influence de l'entre-nœud précédent (soit l'hypothèse $d=0$ ), l'indépendance entre racines d'un même entre-nœud connaissant l'entre-nœud précédent $(b=0)$, ainsi que l'égalité des paramètres des différents entre-nœuds. Cette dernière hypothèse correspondrait au cas où tous les entre-nœuds se comporteraient de façon similaire.

\section{Simulation}

Le modèle permet d'analyser les relations entre racines au travers des paramètres estimés. Par contre, les lois globales, si elles s'expriment facilement formellement, sont difficiles à calculer directement. On peút citer par exemple la distribution du nombre de racines par entre-nœud. Celles-ci peuvent toutefois être obtenues par simulation. Elles nous permettront alors de valider notre modèle et d'en situer les limites en confrontant les résultats de simulation à ceux estimés directement sur nos données.

Les simulations sont effectuées en estimant successivement le nombre et la position des racines à 
chaque entre-nœud. Dans un premier temps, on tire le nombre $I_{2}$ de racines du premier entre-nœud à partir de la probabilité :

$$
P\left(I_{2}\right)=\frac{\int_{\lambda_{2,2} \in[0,2 \pi]} \cdots \int_{\lambda_{2, I_{2}} \in\left[\lambda_{2, I_{2}-1}, 2 \pi\right]} e^{W\left(R_{2}\right)} d \lambda_{2,2} \ldots d \lambda_{2, I_{2}}}{\sum_{I_{2}=1}^{\infty} \int_{\lambda_{2,2} \in[0,2 \pi]} \cdots \int_{\lambda_{2, I_{2}} \in\left[\lambda_{\left.2, I_{2}-1,2 \pi\right]}\right.} e^{W\left(R_{2}\right)} d \lambda_{2,2} \ldots d \lambda_{2, I_{2}}}
$$

La première racine est positionnée à 0 , puis les $12-1$ racines suivantes sont tirées selon la loi de probabilité :

$$
P\left(\lambda_{2, k} / \lambda_{2,2}, . . \lambda_{2, k-1}\right)=\frac{\int_{\lambda_{2, k+1} \in\left[\lambda_{2, k}, 2 \pi\right]} \ldots \int_{\lambda_{2, I_{2}} \in\left[\lambda_{\left.2, I_{2}-1,2 \pi\right]}\right.} \exp \left(W\left(R_{2}\right)\right) d \lambda_{2, k+1} \ldots d \lambda_{2, I_{2}}}{\int_{\lambda_{2, k} \in\left[\lambda_{2, k-1}, 2 \pi\right]} \cdots \int_{\lambda_{2, I_{2}} \in\left[\lambda_{\left.2, I_{2}-1,2 \pi\right]}\right.} \exp \left(W\left(R_{2}\right)\right) d \lambda_{2, k \ldots d \lambda_{2, I_{2}}}}
$$

Une fois complété l'entre-nœud $p$, le suivant est obtenu en simulant dans un premier temps le nombre de racines selon la formule :

$$
P\left(I_{p} / R_{p-1}\right)=\frac{\int_{\lambda_{p, 1} \in[0,2 \pi]} \cdots \int_{\lambda_{p, I_{p}} \in\left[\lambda_{p}, I_{p}-1,2 \pi\right]} \exp \left(W\left(R_{p}\right)\right) d \lambda_{p, 1} \ldots d \lambda_{p, I_{p}}}{\sum_{I_{p}=0}^{\infty} \int_{\lambda_{p, 1} \in[0,2 \pi]} \cdots \int_{\lambda_{p, I_{p}} \in\left[\lambda_{\left.p, I_{p}-1,2 \pi\right]}\right.} \exp \left(W\left(R_{p}\right)\right) d \lambda_{p, 1} \ldots d \lambda_{p, I_{p}}}
$$

qui, par rapport à la formule correspondante donnant $P\left(I_{2}\right)$, permet de prendre en compte l'état de l'entre-nceud précédent. Ensuite, les différentes racines sont simulées de façon similaire à l'entre-nœud 2 à partir de la probabilité :

$$
P\left(\lambda_{p, k} / \lambda_{p, 1}, . . \lambda_{p, k-1}\right)=\frac{\int_{\lambda_{p, k+1} \in\left[u_{p, k}, 2 \pi\right]} \cdots \int_{\lambda_{p, I_{p}} \in\left[\lambda_{\left.p, I_{p}-1,2 \pi\right]}\right.} \exp \left(W\left(R_{p}\right)\right) d \lambda_{p, k+1} \ldots d \lambda_{p, I_{p}}}{\int_{\lambda_{p, k} \in\left[u_{p, k-1}, 2 \pi\right]} \cdots \int_{\lambda_{p, I_{p}} \in\left[\lambda_{p, I_{p}-1}, 2 \pi\right]} \exp \left(W\left(R_{p}\right)\right) d \lambda_{p, k} \ldots d \lambda_{p, I_{p}}}
$$

\section{APPLICATION}

\section{Estimation}

Les estimations et les écarts types des paramètres du modèle général sont présentés dans le tableau I. Les écarts types ont été estimés par inversion de la matrice d'information empirique. On peut y noter une bonne précision des estimateurs, due au nombre de données présentes (63 pivots et 5 entre-nœuds). II n'apparaît pas de relation particulière entre le paramètre $e$ et le niveau de l'entre-nœud. Par contre on observe, pour l'ensemble des entre-nœuds 2 à 5 , une portée de l'interaction entre racines d'un même ni- veau de l'ordre de $90^{\circ}$ correspondant à une valeur de $a=1,5 \mathrm{rd}$. La position d'une racine donnée est donc liée à celles de l'ensemble des racines du même entre-nœud situées sur une demi-circonférence du pied. La portée chute à un quart de circonférence environ pour $E_{6}$. La portée de l'interaction entre deux racines situées sur deux niveaux successifs est du quart de la circonférence pour $E_{3}$ et $E_{4}(c=1,40 \mathrm{rd})$, puis chute avec $E_{5}$ et $E_{6}$

L'intensité de ces interactions ne varie pas aussi régulièrement avec le niveau de l'entrenœud. Elle est plus importante au niveau de $E_{4}$ ( $b=-4,39)$, et à un degré moindre au niveau de $E_{2}(b=-3,43)$.

Tableau I. Estimations et écarts types des paramètres du modèle.

\begin{tabular}{ccccrc} 
Entre-nœud & $a$ & $c$ & $b$ & $d$ & $e$ \\
\hline$E_{2}$ & $1,56(0,19)$ & - & $-3,43(0,14)$ & - & $2,66(0,09)$ \\
$E_{3}$ & $1,58(0,10)$ & $1,40(0,05)$ & $-2,44(0,06)$ & $-1,98(0,05)$ & $2,99(0,04)$ \\
$E_{4}$ & $1,79(0,05)$ & $1,40(0,02)$ & $-4,39(0,04)$ & $-2,05(0,04)$ & $4,59(0,04)$ \\
$E_{5}$ & $1,69(0,04)$ & $1,19(0,03)$ & $-2,84(0,04)$ & $-2,02(0,03)$ & $3,69(0,06)$ \\
$E_{6}$ & $0,88(0,01)$ & $0,56(0,02)$ & $-2,32(0,02)$ & $-1,48(0,03)$ & $3,19(0,02)$
\end{tabular}




\section{Tests d'hypothèses}

L'ensemble des tests effectués et les résultats correspondants sont présentés dans le tableau II. L'hypothèse d'indépendance entre les entrenœuds $(d=0)$ est fortement rejetée avec $\chi^{2}=$ 631,68 pour $8 \mathrm{ddl}$. La contribution au $\chi^{2}$ des différents entre-nœuds est globalement du même ordre de grandeur, même si $E_{4}$ et $E_{5}$ y contribuent un peu plus :

$$
\begin{array}{cccc}
E_{3} & E_{4} & E_{5} & E_{6} \\
121,00 & 185,22 & 183,16 & 142,30
\end{array}
$$

L'hypothèse selon laquelle le nombre et la position des racines sur un entre-nœud ne dépendraient que de l'entre-nœud précédent $(b=0)$ est également rejetée avec $\chi^{2}=1783,70$ pour 8 ddl. Si toutes les différences sont nettement plus importantes que dans le cas précédent, on observe un rejet de plus en plus important avec le niveau de l'entre-nœud considéré :

$$
\begin{array}{cccc}
E_{3} & E_{4} & E_{5} & E_{6} \\
124,98 & 498,42 & 442,22 & 718,28
\end{array}
$$

Il existe donc des dépendances dans la position et le nombre de racines à l'intérieur d'un entre-nœud comme entre entre-nœuds succes sifs.

L'hypothèse d'égalité des paramètres correspondant aux liaisons entre racines d'un même entre-nœud et entre racines de 2 entre-nœuds successifs est également rejetée avec $\chi^{2}=$ 222,72 pour 8 ddl qui se décompose ainsi par entre-nœud :

$$
\begin{array}{cccc}
E_{3} & E_{4} & E_{5} & E_{6} \\
11,08 & 100,36 & 34,52 & 76,76
\end{array}
$$

Tous les niveaux contribuent au $\chi^{2}$, mais les valeurs sont beaucoup plus faibles que les pré-

\begin{tabular}{|c|c|c|c|}
\hline Hypothèse & $d d l$ & $x^{2}$ & seuil (5\%) \\
\hline$d=0$ & 8 & 631,68 & 15,5 \\
\hline$b=0$ & 8 & 1783,70 & 15,5 \\
\hline$a=c b=d$ & 8 & 222,72 & 15,5 \\
\hline égalité des entre-nœuds & 15 & 1934,34 & 25,0 \\
\hline$c=\alpha a ; d=\alpha b$ & 4 & 10,88 & 9,5 \\
\hline
\end{tabular}
cédentes. De plus, l'hypothèse de constance des paramètres sur l'ensemble des entre-nœuds,

Tableau II. Tests de différentes hypothèses. testée en supprimant le premier entre-nœud est rejetée avec $\chi^{2}=1934,34$ pour $15 \mathrm{ddl}$.

Un dernier sous-modèle testé suppose simplement la proportionnalité des paramètres jouant le même rôle soit $c=\alpha a$ et $d=\alpha b$. Il est également rejeté avec $\chi^{2}=10,88$ pour 4 ddl qui se décompose en :

$$
\begin{array}{cccc}
E_{3} & E_{4} & E_{5} & E_{6} \\
8,54 & 1,36 & 0,00 & 0,58
\end{array}
$$

Toutefois, on peut remarquer que ce modèle n'est rejeté qu'au niveau de $E_{3}$. De plus il permet d'évaluer l'importance respective de l'entre-nœud précédent dans l'établissement des racines : le paramètre de proportionnalité $(\alpha)$ chute régulièrement avec le numéro de l'entre-nœud :

$$
\begin{array}{cccc}
E_{3} & E_{4} & E_{5} & E_{6} \\
0,84 & 0,75 & 0,71 & 0,70
\end{array}
$$

Les racines adventives du maïs sont donc des racines aux dispositions très structurées les unes par rapport aux autres. Si la liaison est très importante entre racines d'un même niveau, la liaison avec celles de l'entre-nœud précédent n'est pas négligeable, de l'ordre de $75 \%$ de la précédente.

\section{Validation}

\section{Nombre de racines par entre-ncud}

Le tableau III donne la moyenne et l'écart type du nombre de racines des populations mesurées sur le terrain et estimées. On peut noter un bon accord entre moyennes avec un écart de moins de $10 \%$. II en est de même des écarts types qui sont du même ordre de grandeur dans les 2 cas. La comparaison directe entre histogrammes (fig 3) montre que le modèle fournit des distributions très proches de celles obtenues sur les données observées. Les $\chi^{2}$ d'ajustement entre les 2 distributions, présentés dans la dernière colonne du tableau III confirment cette observation. Le modèle conduit simplement à une gamme de variation légèrement plus large, comme par exemple au niveau $E_{2}$ où il génère des pieds avec $I_{2}=1$, avec une probabilité de $0,38 \%$. II lisse également légèrement les données avec par exemple un pic à $64,66 \%$ pour $I_{4}$ dans les simulés contre $71,43 \%$ dans les observés.

Une analyse statistique des histogrammes croisés est impossible, vu le nombre de pieds 
Tableau III. Statistiques des distributions du nombre de racines observées et simulées par entre-nœud.

\begin{tabular}{|c|c|c|c|c|c|c|c|}
\hline \multirow[b]{2}{*}{ Entre-nœud } & \multicolumn{2}{|c|}{ Observés } & \multicolumn{2}{|c|}{ Simulés } & \multirow[b]{2}{*}{$\chi^{2}$} & \multirow[b]{2}{*}{$d d l$} & \multirow[b]{2}{*}{ seuil ( $5 \%)$} \\
\hline & Moyenne & Ecart type & Moyenne & Écart type & & & \\
\hline$E_{2}$ & 3,43 & 0,61 & 3,40 & 0,72 & 2,33 & 5 & 11,1 \\
\hline$E_{3}$ & 1,89 & 0,72 & 1,84 & 0,65 & 2,71 & 5 & 11,1 \\
\hline$E_{4}$ & 2,95 & 0,58 & 2,95 & 0,63 & 4,96 & 4 & 9,5 \\
\hline$E_{5}$ & 2,62 & 0,66 & 2,55 & 0,71 & 1,16 & 4 & 9,5 \\
\hline$E_{6}$ & 5,43 & 1,17 & 5,39 & 1,11 & 4,63 & 7 & 14,1 \\
\hline
\end{tabular}

observés, conduisant à un trop faible effectif par case. Toutefois, on peut noter sur les corrélations entre $I_{n}$ et $I_{n-1}$ pour les données mesurées et simulées (données dans le tableau IV) que le modèle rend compte de la corrélation négative entre 2 entre-nœuds donnés et en respecte les ordres de grandeur.

\section{Position des racines}

L'observation des positions des racines sur un entre-nœud donné est faite au travers des histogrammes des angles entres racines successives, le pied étant observé dans le sens trigonométrique. Ces angles dépendent à la fois de l'entrenœud et du nombre de racines, l'analyse doit donc être faite conditionnellement à chacun des critères. Plusieurs situations pouvant être issues d'un même pivot, les données dont proviennent ces histogrammes ne sont pas indépendantes.
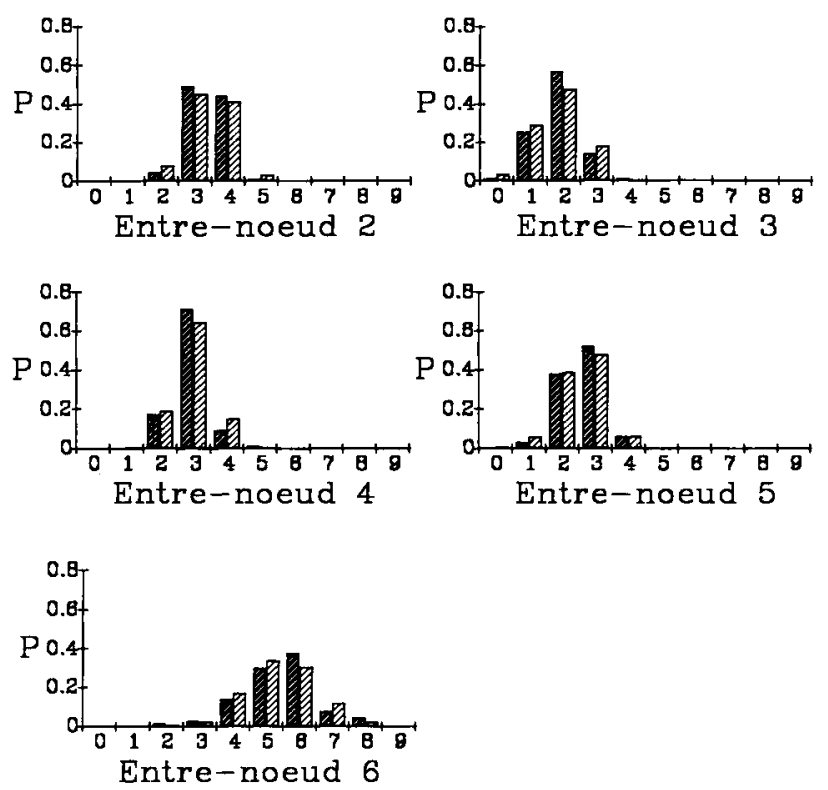

Fig 3. Histogramme du nombre de racines par entre-nceud (population observée en foncé, simulée en clair).
On ne peut donc pas les comparer par test du $\chi^{2}$. De ce fait, seule l'allure générale de ces histogrammes est analysée. La figure 4 donne les résultats obtenus sur pieds simulés et observés en détaillant par entre-nœud, la figure 5 en détaillant par entre-nœud et par nombre de racines. Nous n'avons retenu dans l'analyse que les cas où plus de 50 angles étaient présents pour assurer une certaine stabilité des courbes obtenues. De ce fait, tous les cas possibles ne peuvent être abordés de par la faiblesse de l'échantillon dans certains cas. Globalement, on note des histogrammes beaucoup plus réguliers dans le cas des pieds simulés. Au-delà de cette remarque globale, on peut noter un bon accord entre observés et simulés, mis à part le cas de l'entre-nœud 3. On peut également noter en effet que les pics sont plus marqués au niveau des observés dans les cas $I_{2}=3$, et $I_{3}=2$. Dans ce dernier cas, on observe deux pics à $120^{\circ}$ et $240^{\circ}$ correspondant à l'influence de l'entre-nœud précédent, mais il est plus marqué au niveau des racines observés.

Pour comparer la distribution des racines de $E_{i+1}$ dans les angles définis par les racines de $E_{i}$

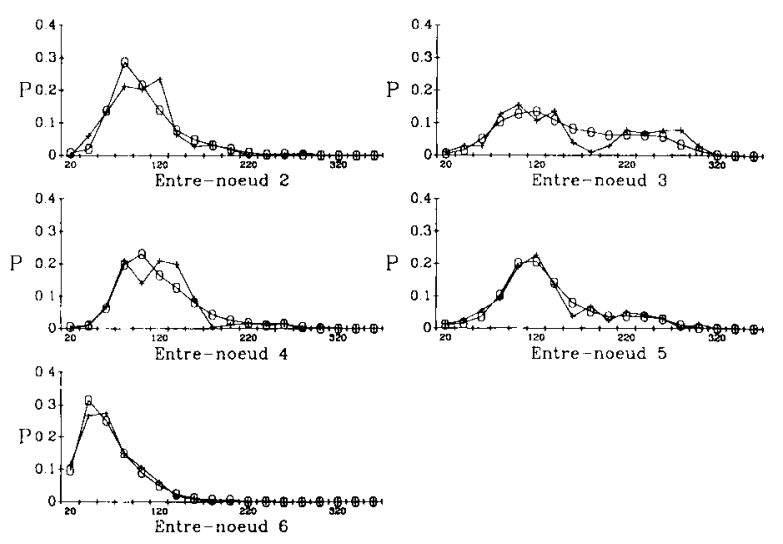

Fig 4. Probabilité d'observer un angle donné entre 2 racines consécutives sur un entre-nœud donné (population observée $\rightarrow-$ simulée $-0-$ ). 
Tableau IV. Matrices de corrélation du nombre de racines par entre-nœud.

\begin{tabular}{|c|c|c|c|c|c|c|c|c|c|c|}
\hline & \multicolumn{5}{|c|}{ Observés } & \multicolumn{5}{|c|}{ Simulés } \\
\hline$E_{2}$ & $\begin{array}{r}E_{2} \\
1,00\end{array}$ & $\begin{array}{c}E_{3} \\
-0,36\end{array}$ & $\begin{array}{c}E_{4} \\
0,15\end{array}$ & $\begin{array}{c}E_{5} \\
-0,23\end{array}$ & $\begin{array}{c}E_{6} \\
0,01\end{array}$ & $\begin{array}{c}E_{2} \\
1,00\end{array}$ & $\begin{array}{c}E_{3} \\
-0,29\end{array}$ & $\begin{array}{c}E_{4} \\
0,09\end{array}$ & $\begin{array}{c}E_{5} \\
0,04\end{array}$ & $\begin{array}{c}E_{6} \\
0,00\end{array}$ \\
\hline$E_{3}$ & $-0,36$ & 1,00 & $-0,24$ & 0,31 & $-0,02$ & $-0,29$ & 1,00 & $-0,39$ & 0,11 & 0,02 \\
\hline$E_{4}$ & 0,15 & $-0,24$ & 1,00 & $-0,47$ & 0,31 & 0,09 & $-0,39$ & 1,00 & $-0,30$ & 0,04 \\
\hline$E_{5}$ & $-0,23$ & 0,31 & $-0,47$ & 1,00 & $-0,31$ & 0,04 & 0,11 & $-0,30$ & 1,00 & $-0,19$ \\
\hline$E_{6}$ & 0,01 & $-0,02$ & 0,31 & $-0,31$ & 1,00 & 0,00 & 0,02 & 0,04 & $-0,19$ & 1,00 \\
\hline
\end{tabular}

nous avons repris la méthode proposée par (Pellerin et al, 1989). Soient $R_{i, k}$ et $R_{i, k+1}, 2$ racines successives de $E_{i}$ mesurées dans le sens trigonométrique, et $V_{i, k}$ la bissectrice de l'angle ainsi défini. Soit $\beta$ l'angle défini par $R_{i, k}$ et $R_{i, k+1}$ et $\alpha$ l'angle entre une racine de $E_{i+1}$ tombant dans $\beta$ et la bissectrice. Nous traçons l'histogramme du rapport $\alpha / \beta$, c'est-à-dire de la position relative de la racine de l'entre-nœud supérieur dans l'angle $\beta$. Ceci a été fait dans les cas où 1 ou 2 racines de $E_{i+1}$ sont présentes dans l'angle $\beta$ (fig 6 ).

Dans le cas où une seule racine est présente sur l'entre-nœud $k+1$, on retrouve l'existence d'un mode centre sur 0 correspondant à une disposition à égale distance des 2 racines de l'entre-nœud précédent. Cette disposition centrée sur 0 correspond en fait à la position de potentiel maximal de la racine $E_{i+1}$ dans l'angle formé par les 2 racines $R_{i, k}$ et $R_{i, k+1}$.

Dans le cas à 2 racines, on observe deux modes relativement proches de $-1 / 6$ et $1 / 6$. Seul le cas de l'entre-nœud 2 présente un écart entre distributions observées et simulées. Cette disposition correspond là aussi à la maximisation du potentiel maximal des 2 racines de $E_{i+1}$ dans
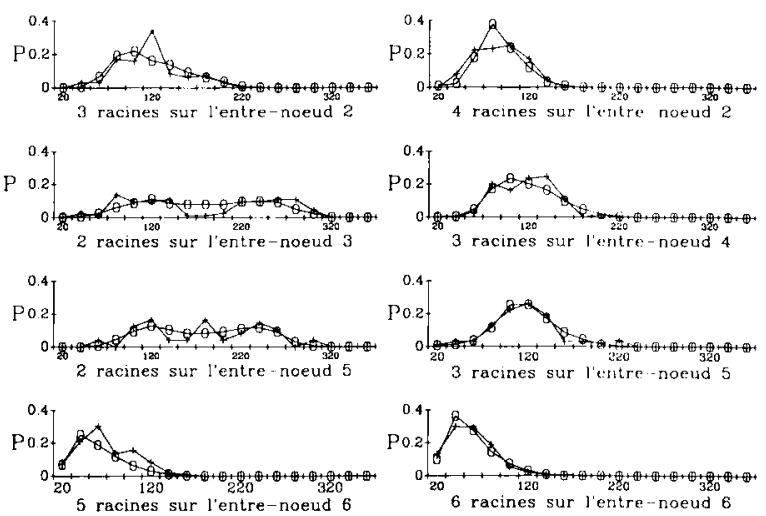

Fig 5. Probabilité d'observer un angle donné entre 2 racines consécutives sur un entre-nœud donné pour différents nombres de racines sur l'entre-nceud (population observée $\longrightarrow$, simulée $\longrightarrow$ - l'angle formé par $R_{i, k}$ et $R_{i, k+1}$. Toutefois, les autres racines de ces 2 entre-nœuds interviennent également dans le potentiel total, et donc dans la position de ces racines, mais à un degré moindre du fait de la décroissance du potentiel entre deux racines avec l'angle qui les sépare.

\section{CONCLUSION}

Le modèle proposé de description de la disposition des racines primaires du maïs autour de la tige rend relativement bien compte de la structure des pieds observés. II rend bien compte en particulier de la disposition régulière des racines sur un entre-nœud donné et de la disposition alternée des racines sur 2 entre-nœuds successifs. II permet, au travers des paramètres estimés, de quantifier globalement et de tester les relations entre positions des racines à la fois sur un même entre-nœud et sur 2 entre-nœuds successifs. Ainsi l'interaction entre 2 racines situées sur 2 entre-nœuds successifs est d'environ $75 \%$ de celle entre 2 racines d'un même entre-nœud, tant en intensité maximale qu'en portée. Cette in-
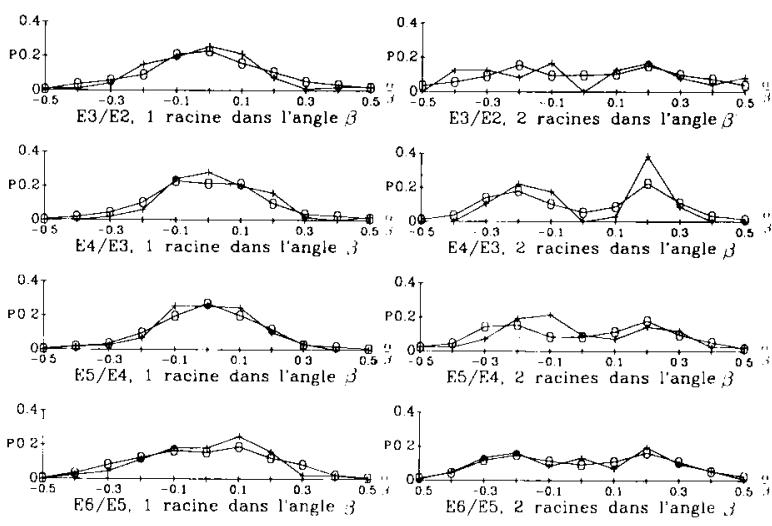

Fig 6. Probabilité d'observer le rapport $\alpha / \beta$ où $\beta$ représente le demi-angle entre 2 racines consécutives de $E_{i}$ et $\alpha$, l'angle entre la bissectrice de ces racines et les racines de $E_{i+1}$ qui tombent dans l'angle $2 \beta$. Cas où 1 ou 2 racines de $E_{i+1}$ tombent dans $2 \beta$ (population observée -+ , simulée $\multimap-$ ) 
teraction entre racines de 2 entre-nœuds successifs décroit avec l'ordre des entre-nœuds. Ce résultat est à rapprocher de l'observation morphologique des pieds qui montre que les entrenœuds de rang élevé sont plus allongés. Les racines que portent 2 entre-nœuds successifs sont donc plus éloignées le long de la tige, ce qui pourrait expliquer la plus grande indépendance constatée entre leurs positions relatives.

Dans le modèle actuel, la fonction choisie pour décrire le potentiel d'interaction est un compromis entre la forme de l'interaction attendue et la facilité de calcul et d'estimation. Dans un souci d'amélioration du modèle, d'autres fonctions pourraient être testées. De même, l'existence d'interactions avec d'autres organes portés par la tige, les bourgeons axillaires en particulier, devrait être testée. L'existence de telles interactions est en effet suggérée par l'observation de certains pieds.

Tel qu'il est, le modèle proposé est cependant intégrable à un modèle général de simulation de l'architecture racinaire du maïs. L'étude des trajectoires des racines primaires dans le sol réalisée par ailleurs a montré qu'en l'absence d'obstacle mécanique de grande dimension, chaque racine restait dans un même plan vertical défini par l'angle d'émission de la racine autour de la tige (Tardieu et Pellerin, 1990). L'utilisation combinée du modèle proposé dans cet article et des résultats acquis relatifs aux trajectoires racinaires devrait permettre de simuler en 3 dimensions l'architecture du système racinaire primaire et de générer des cartes d'impacts racinaires simulées dans des plans horizontaux qui pourront être comparées à des cartes observées.

L'étude de la disposition des organes chez les végétaux a fait l'objet de nombreux autres travaux. Les résultats existants concernent surtout la disposition des organes aériens autour de la tige, les données relatives au système racinaire étant beaucoup plus rares. II existe cependant des travaux relatifs à la disposition des racines secondaires sur les primaires (Bell et McCully, 1970). Chez le maïs, les racines secondaires apparaissent à partir de cellules du péricycle situées en face des pôles de xylème. Dans ce cas leur disposition est définie par la structure vasculaire de la racine primaire et le processus de division cellulaire. Dans le cas de la disposition des racines primaires autour de la tige, on a vu que les observations histologiques disponibles ne permettent pas de retenir cette explication. Une autre hypothèse parfois avancée pour expliquer l'existence de dispositions particulières est qu'il existerait autour d'un primordium d'organe une zone d'inhibition hormonale empêchant l'apparition d'un autre primordium dans son voisinage immédiat (Jean, 1983). Le modèle proposé dans cet article est compatible avec une hypothèse de ce type. La mise en évidence du mécanisme réellement en jeu générant les dispositions observées des racines autour de la tige nécessiterait cependant une étude physiologique et histologique plus fine de leur ontogenèse.

\section{RÉFÉRENCES}

Bell JK, McCully ME (1970) A Histological Study of Lateral Root Initiation and Development in Zea mays. Protoplasma 70, 179-205

Dacunha-Castelle D, Duflo M (1983) Probabilités et statistiques. 2. Problèmes à temps mobile. Masson, Paris

Diggle AJ (1988) Rootmap - a model in threedimensional coordinates of the growth and structure of fibrous root systems. Plant Soil 105, 169178

Girardin P, Jordan MO, Picard D, Trendel R (1986) Harmonisation des notations concernant la description morphologique d'un pied de maïs (Zea mays L). Agronomie 6, 873-875

Girardin P, Morel-Fourrier B, Jordan MO, Millet B (1987) Développement des racines adventives chez le maïs. Agronomie 7, 353-360

Hoppe DC, McCully ME, Wenzel CL (1987) The nodal roots of Zea: their development in relation to the structural features of the stem. Can J Bot 64, 25242537

Jean RV (1983) Introductory Review, Mathematical Modeling in Phyllotaxis: The State of the Art. Math Biosci 64, 1-27

Lungley DR (1973) The growth of root systems. A numerical computer simulation model. Plant Soil 38, 145-159

Pages L, Aries F (1988) Sarah : modèle de simulation de la croissance, du développement et de l'architecture des systèmes racinaires. Agronomie 8, 889-896

Pellerin S, Tardieu F, Tricot F (1988) Modelling the root system architecture: experimental data on maize root system geometry. ISRR Symp on plant roots and their environment, 21-26 août 1988 Uppsala, Suède 
Pellerin S, Tricot F, Chadœuf J (1989) Disposition des racines adventives autour de la tige de maîs (Zea mays L). Agronomie 9, 859-866

Picard D, Jordan MO, Trendel R (1985) Rythme d'apparition des racines primaires du maîs (Zea mays L). I. Etude détaillée pour une variété en un lieu donné. Agronomie 5, 667-676
Stoyan D, Kendall WS, Mecke J (1987) Stochastic Geometry and Its Applications. J Wiley \& Sons, Akademie-Verlag, Chichester, Berlin

Tardieu F, Pellerin S (1990) Trajectory of the primary roots of maize in fields with low mechanical constraints. Plant Soil 124, 39-45 